\title{
Usability Readiness of German Software SMEs - Three Segments and their Characteristics
}

\author{
Karl Werder ${ }^{2}$, Phillip Haake ${ }^{2}$, Alexander Maedche $e^{1,2}$ \\ Chair of Information Systems IV, University of Mannheim, http://eris.bwl.uni-mannheim.de ${ }^{1}$ \\ Institute for Enterprise Systems, University of Mannheim, http://ines.uni-mannheim.de ${ }^{2}$
}

\begin{abstract}
German small-and-medium sized software companies are facing an increasing pressure by their users with regards to usability expectations. While some software companies still heavily emphasize functional aspects of their products, others have identified the potential of usability to differentiate themselves. The study has an exploratory nature and employs cluster analysis to investigate responses of German SME software companies with a specific focus on usability. We identify three segments of companies, i.e. Usability Sleeper, Usability Executers and Usability Performers. While the first perform worst, the Usability Executers show high usability readiness with regard to software development (SD) processes. Usability Performers further separate themselves through higher employees' expertise. Subsequent analysis of these sub-segments reveals that business sector and management support are key differentiators amongst them. For practitioners the results presented in this paper describe the characteristics of SME software companies in Germany with mature usability.
\end{abstract}

\section{Introduction}

Small and medium-sized enterprises (SMEs) in Germany are often highly specialized firms and known to be the drivers for innovation in the German economy (Simon 2009). The same holds true for the German software industry. In addition, software companies are facing multiple challenges, e.g. cost pressures, increasing global competition and growing expectations of users (Woywode et al. 2012). More recently, software firms are facing endusers that are surrounded by easy-to-use software-based solutions in their private life. Thus, they begin to expect a comparable usability of the software solutions at their workplace. This demand for usability should not only be considered a threat, but as an opportunity to seek competitive advantage (Gemser et al.2006). Especially, software firms in the US have been 
able to develop software products with superior usability and leverage their usability knowhow for achieving such advantages (Scheiber et al. 2012). As a result, the German federal ministry for economics and technology initiated the execution of a comprehensive empirical study in 2011 (UIG study) to explore the status quo of usability methods in German SMEs (Woywode et al. 2012). The study was designed to capture the status quo of German SMEs in the software industry and user organizations with regard to the knowledge and actual use of usability concepts. In part, it was concluded that there is still a large gap between US and German software firms in the adoption of usability practices and knowledge. Usability is an important factor that influences the success of SD efforts and is therefore often used to measure software product quality (Madan \& Dubey 2012). Hence, this paper specifically investigates possible segments focusing on the usability readiness of German SMEs. A segmentation of the responses to the software company questionnaire of the UIG study is presented. Such an approach allows to answer the following two research questions: Do different segments with regard to usability readiness in German software SMEs exist? What are the characteristics of these segments?

The remainder of this paper will first present related work to the study. In section 3 , the research approach including data collection and analysis will be presented. Section 4, includes a description of the results focusing on the identified dimensions and segments. The discussion in section 5 is used to explain the results of the research and to put them into context. The paper ends with a conclusion, the limitations of the study.

\section{Related Work}

The German software business environment can be separated in a primary and a secondary sector, which have been described by Friedewald et al. (2001). Most firms in the primary sector (e.g. data processing firms) specialize in SD and sales. They usually have less than ten employees. This sector of the German software industry is dominated by SAP (Brault et al. 2013). However, many smaller players thrive in the largest European software market (Brault et al. 2013). The secondary sector is dominated by larger firms that develop software, which is included in their products or accompanies them (Friedewald et al. 2001). Since this is study is based on the UIG-study (Woywode et al. 2012) we use the same EU - definition to categorize primary sector software SMEs. Accordingly, companies with an annual turnover below 50 million Euros and less than 250 employees are defined as SMEs (European Union Commission 2003).

Usability is a socio-material concept (Riemer \& Vehring 2010) and a very important characteristics of software products. They need to be developed with stringent methods (Madan \& Dubey 2012). Prior research already indicated the need for training and education of developments team to achieve significant improvements with regard to usability (Seffah \& Metzker 2004). Nonetheless, various definitions for usability exist (cf. Madan \& Dubey 2012). For the purpose of this research we adopt the ISO standard (International Organization for Standardization 1998).There is little research on the aspect of usability methods for small and 
medium sized SD organizations (Woywode et al. 2012). We add to this research by exploring the influencing factors for the incorporation of usability methods in such firms.

\section{Research Method}

\subsection{Data Collection}

Specific emphasis was set to collect a representative sample of SMEs in Germany to allow for generalization. We collected data from software companies with 6-10 employees on average. The sample representing software companies covered 1.756 companies with 208 respondents, i.e. a response rate of $11.85 \%$. The data collection was conducted from January to November 2011. Initially, the software companies were approached via postal mail with an option to complete the questionnaire electronically. A unique identifier assured that each response was given by companies from the defined sample. Three days after postal delivery, the companies were approached via phone to increase the response rate. The questionnaire covered various aspects of usability affecting the company on an organizational and product level. On the organizational level questions related to the performance and environment of the company, employee selection and expertise, usability self-assessment, knowledge sources and knowledge exchange with environment, customers and users, and general information about the company. On the product level information about the SD process with a specific focus on usability aspects were gathered. The questionnaire contained 54 mandatory questions and 83 items. All items used and described below were measured on a 7-point Likert scale ${ }^{1}$. Moreover, the questionnaire gathered further optional information on the individual and company level.

In the following we present only the parts of the survey that are relevant to answering our research question. With regard to the SD process we focused on the following categories on the product level: the usage of usability methods, the actual UI development process and the inclusion of users into the development process. Furthermore, we first looked at employee expertise, which includes the different roles that are trained on the subject of usability, whether an employee willing to speak at a convention about usability methods could be identified, and the self-assessment along the two categories, i.e. the usability rating of the own software products and the general attitude of management towards usability. The questionnaire also assessed the contribution of individual customer segments to overall turnover and the importance of trends within the next 5 years. Companies' development in the last 3 years was assessed along key performance indicators, i.e. turnover, number of employees, customer satisfaction and profit.

\subsection{Data Analysis}

For the statistical analysis we employed SPSS v22. We started analyzing the dataset of software companies using exploratory factor analysis. However, SPSS was unsuccessful in

\footnotetext{
${ }^{1}$ The entire questionnaire is publicly accessible in Woywode et al. (2012)
} 
calculating the correlation matrix for all items, as it was not found to be positive definite. When reducing the included items to focus on a subsection of the questionnaire, it became possible to calculate the correlation matrix, but we were not able to find a combination that leads to a determinant that is high enough to rule out any multicollinearity issues. Therefore, we opted for an alternative approach, identifying dimensions of thematically related questions in the questionnaire. Therefore, for the remaining 49 items we calculated the Cronbach's alpha for those items contributing to a dimension. Cronbach's alpha $\alpha$ is a reliability measure, whereas $\mathrm{N}$ refers to the number of items used to build the dimensions.

Following the successful identification of dimensions we used this angle to determine possible segments in a two-step process. Through the exploratory nature of this research, we first investigated the identification of possible segments via the use of hierarchical cluster analysis (Kachigan 1986). As input for clustering we selected the dimension related to the user-centered software development process (UCSDP) readiness, whereas we define readiness as the adoption level of usability methods, techniques and practices. We ground this decision in the fact that the development process readiness is a key factor influencing the product quality (Harter et al. 2000). Using between groups linkage, i.e. the mean distance of all pairs across two segments, lead to the creation of two segments. Once we identified the existence of the two segments, we created new items segmenting the two using the K-means algorithm (Mackay 2003). Subsequently, we employed crosstabs and independent sample t-test to better understand the characteristics of the identified segments and possible contrasts within the segments. Preliminary results were discussed with four IS experts indicating further differentiating factors.

\section{Results}

\subsection{Descriptive Results - Key Dimensions}

User-Centered Software Development Process (UCSDP): The first dimension looks at the

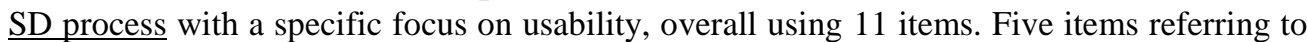
usability methods measured the insensitivity of use of 1) methods for user analysis (e.g. contextual interviewing, scenarios, user profiles), 2) design methods (e.g. wireframes, prototyping), 3) usability evaluation methods (e.g. user testing, heuristics / guidelines), 4) specialized usability software (e.g. Balsamiq), and 5) whether such methods are already applied for a long time. Two items measured the development process for the user interface, i.e. the design of the UI before the development activities and the use of style guides for the development activities. Furthermore, four items measured the inclusion of users in the SD process. The items asked whether the users are interviewed 1) about typical tasks that the software is going to be used for (e.g. use cases, scenarios), 2) to generate user profiles, and 3) to assess first mock-ups of the user interface. Furthermore, it was assessed whether 4) the software is always tested on usability aspects before releasing the product into the market. We can summarize all items related to the development process to a reliable dimension looking at the entire UCSDP $($ Cronbach's alpha $(\alpha)=0.864$; Number of items $(\mathrm{N})=11$ ). 
Management Assessment. To better understand the management perspective of usability subjects, we also asked questions related to their support and understanding of such topics. Items included whether management a) sees the need to support usability on an ongoing basis, b) is enthusiastic about opportunities offered through usability, c) invests time required to understand how the companies can profit from usability, d) decided to prioritize usability aspects over functional aspects, e) defined clear usability goals, and f) supports the achievement of usability goals. The aforementioned items lead to a reliable dimension of perceived management support $(\alpha=0.928 ; \mathrm{N}=6)$. Besides questions related to specific characteristics of the organization, we also asked firms to self-assess the evolution of their company throughout the past 3 years. The items included the development of the turnover, number of employees, customer satisfaction, and profit. Moreover, we asked to self-evaluate the development of their turnover, number of employees and profit related to their industry's average, and their overall impression of the companies development over the last 3 years. We can summarize these items into a reliable dimension measuring the companies development over the last 3 years $(\alpha=0.891 ; \mathrm{N}=8)$.

Human Resources. We relied on different items about employee skills relevant for the recruitment of new hires to construct the employee recruitment dimension $(\alpha=0.763 ; \mathrm{N}=6)$. All items are measured from unimportant to very important. Six skills or knowledge areas had to be assessed, i.e. programming, design and presentation, usability, psychology, economics, and business informatics. Measuring the training efforts of the firms lead to a highly reliable dimension $(\alpha=0.861 ; \mathrm{N}=5)$. The dimension assessed the degree of decision authority of employees with usability responsibility, whether developers, usability employees, or employee affected by the SD process are trained on usability subjects, and whether employees are trained for a long time on usability subjects. All items were measured from strong disagreement to strong agreement on a seven-point Likert scale. Likewise the expertise of employees was measured and resulted in a reliable dimension $(\alpha=0.894 ; \mathrm{N}=3)$. The subjects were confronted with a hypothetical questions, assuming that a large convention invites the company to talk about usability. The questions assessed whether or not the firm has an employee who could be send to the convention, being an expert on method of user research, methods to design an application and usability evaluation methods.

Business Environment. The dimension competitive environment was measured as the development of the firm's environment over the last 3 years and was found to be reliable $(\alpha=0.796 ; \mathrm{N}=5)$. The growth opportunity in the current competitive environment and the rate of innovation in the industry were both measured from dramatically decreasing to dramatically increasing. Furthermore, the change in production and service technologies, and the change of customer needs were both measured from no change to strong change. The research and development activities in the industry were measured from strong decreasing to strong increasing. The contribution of product offerings to the company's turnover was found to be reliable after removing one item, that asked rather openly for the contribution of other services $(\alpha=0.622 ; N=5)$. Assessing the relative importance of the sale of standard software licenses, the implementation of standard software, the development of custom made software, the execution of maintenance and support, and the execution of trainings and instructions was measured from not important to very important. 


\subsection{Segments}

Resulting from the cluster analysis we identify segments in our dataset and describe these in further detail. It is noteworthy, that our list of dimensions is not exhaustive. However, based on the insights of the discussions and our understanding of the underlying data we deem the following dimensions as highly relevant for the context of our investigation. Moreover, further characteristics of such segments are identified and presented below (see also Figure 1).

\section{Usability Sleepers vs Usability Executors}

We found the user-centered development process dimension to be a leading criteria for distinguishing the responses. This higher level dimension is constructed from the dimensions of usability methods, development process and inclusion of user (see Appendix). Using this higher level dimension to build segments, we find a low readiness segment $(\mathrm{M}=2.71 ; \mathrm{SD}=0.84$ $\mathrm{N}=79)$ that is substantially different from the high readiness segment $(\mathrm{M}=4.78 ; \mathrm{SD}=0.73$; $\mathrm{N}=88$ ). Besides the chosen dimension related to the development process, we identified differences in employee related measurement. Using an independent samples t-test to compare the groups along the expertise of employees dimension, finding a highly significant difference $(\mathrm{t}=-8.460 ; \mathrm{p}=.000)$. This means that companies with high UCSDP readiness also feel confident to talk about their expertise in methods of user research, methods to design an application and usability evaluation methods.

\section{A closer look at the Usability Performers segment}

Having gathered knowledge on the differences of the original segments, we turned our interest to those companies that seem to be at the forefront already. Therefore, we had another look at the high readiness segment in particular, trying to find further distinguishing characteristics within this segment of companies. We analyzed the data within the high readiness segment again using an exploratory approach. We started to identify possible sub-segments within the high readiness segment along the expertise dimension. Once we confirmed the existence of two sub-segments we used the k-means clustering algorithm to establish a new variable assigning each dataset to one of the sub-segments. Therefore, establishing a sub-segment with low employee expertise $(\mathrm{M}=2.53 ; \mathrm{SD}=1.38 ; \mathrm{N}=40)$ and a second sub-segment with high employee expertise $(\mathrm{M}=5.14 ; \mathrm{SD}=1.27 ; \mathrm{N}=44)$.

For those companies that have higher employee expertise, we also find a significant difference in the contribution of customers from the retail industry $(t=-2.302 ; p=.024)$. This reflects a higher contribution to the overall turnover from the customer-oriented industry. Also, we find a significant difference in the responses of perceived usability of competitor's products ( $t=-$ 2.259; $\mathrm{p}=.027)$. Moreover, the perceived importance of usability as a trend over the next 5 years shows a significant difference for companies with higher employee expertise $(t=-2.526$; $\mathrm{p}=.014)$. These differences indicate that firms with higher employee expertise on usability also perceive more competitive pressure. We performed independent samples t-test's to analyze the two groups, identifying significant differences in the dimension perceived management support ( $\mathrm{t}=-2.0375 ; \mathrm{p}=.041)$. Management support is an important influencing factor for the direction and priorities of organizations. We find that firms with higher employee expertise with regard to usability also have a management that is more enthusiastic about usability. 
Besides, management also invests more time to understand the benefits of usability to the company as a whole. Moreover, the management in such companies clearly defines usability goals and it assigns higher value to usability than functional aspects of the product. In addition, we find that firms with more expertise on usability also seem to have more customers from the retail industry. Furthermore, these companies perceive more competitive pressure and in turn exhibit more management support on usability matters.

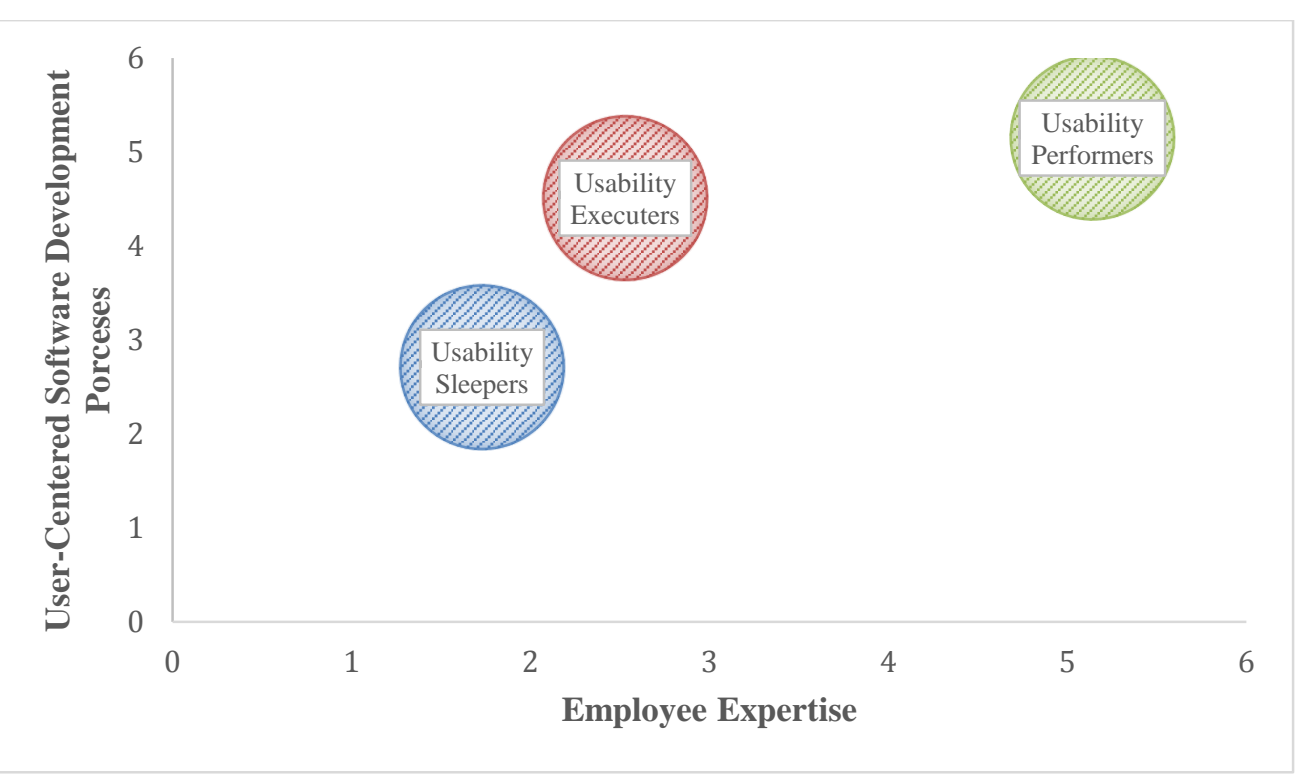

Figure 1 - Visualization of the three identified segments

\section{Discussion}

The thorough analysis of the questionnaire and our exploratory approach allowed us to identify two important differentiators among the high usability readiness firms. First, we will discuss the influence of business sector under consideration of usability in the SD process. Second, the outmost importance of management support for the success of efforts for increased usability will be stressed.

\section{The influence of the business sector on usability readiness}

The business sector seems to have an important moderating role as those software companies which have a high share of revenue from the retail sector also have more usability expertise in their company. This development might be induced by the general development of ecommerce. Usability expertise and even user experience management have progressed much faster in e-commerce as in the traditional enterprise systems realm. Retail firms have to think about the new threats to their business model through the new multi-channel business environment. The retail industry is adapting to new challenges through fierce international 
competition. Such companies (e.g. Amazon) have analyzed usability and user experience of their online presences in great detail. Furthermore, user organizations in the retail industry are close to B2C customers and generally have less specialized staff. These two factors may lead to additional pressure towards usability of enterprise systems in the retail sector. All in all, there are strong indicators that the user organization and their demands act as accelerators for usability developments in the German software industry. SMEs that have a profound knowledge of usability methods also seem to have a significantly different view on the perceived usability of competitors' products. They perceive the products of competitors as to be significantly better in terms of usability than those in the segment with a lower readiness. This shows the great value of usability knowledge as enabler for observing and understanding competitive threats. However, this can also be interpreted as being due to the business sector that the firms are in, since user organizations' expectations seem to fuel the usability efforts of the software SMEs. What is more, the perceived importance of usability as a trend over the next five years also varies significantly between high and low segment firms. Greater knowledge is connected with a higher perceived importance of the usability trend.

\section{The influence of management support on usability readiness}

Management support has long been known to be important for the overall success of information systems projects (Doll 1985). Our exploratory analysis leads to the finding of significantly higher management support for usability initiatives for the sub-segment of firms with a high usability expertise. The enthusiasm of the management about usability seems to be an important driver for gathering usability knowledge in an organization. This finding is further supported by the fact that there is also a significant difference in the reported time that is invested by the management to understand the benefits of usability to the company between the two segments. The prioritization of usability over functional aspects and the definition of clear usability goals was significantly higher for firms in the segment with a very high usability expertise. In turn this means further knowledge with regard to user research, application design, and methods for usability evaluation. Thus, it can be said that management support is crucial for the success of usability initiatives and the general depth of the implementation of usability methods in German software SMEs.

Today, German software SMEs face the growing pressure of end-user expectations in terms of the growing demand for the consumerization of their products (Degarmo et al. 2011; Scheiber et al. 2012). It seems necessary that firms react to this trend or even shape it proactively (e.g. Degarmo et al. 2011). To develop a holistic strategy for implementing usability methods in their SD organizations can be a key mechanism for German Software SME's to deal with competitive pressure arising from the trend of consumerization for endusers. Already, some firms are responding to the growing market pressure by developing a deeper understanding about usability design methods and how they can enable themselves to meet the clients' demands. However, the results of the study indicate that many German software SMEs are still in strategic straightjackets. They are mainly focused on the design and inclusion of new functions into their products (Scheiber et al. 2012) instead of the overall value creation for the end-user and subsequently customer organization. This is a potential impediment to future growth as more and more customer organizations in Germany seem to focus on usability (Scheiber et al. 2012). 


\section{Conclusion}

German software SMEs can be divided into segments of firms that either have a high usability readiness and those that have a significantly lower one. In addition, the segment of high readiness firms can be differentiated even more alongside other characteristics, such as managements' attitude towards usability and the general perception of the business sector. We were able to show that environmental factors seem to influence the absorption of usability methods in the German software industry, which mainly provides highly specialized solutions for specific applications markets. Furthermore, it has been shown that the management's perception is of particular importance for determining the intensity of a firm's usability initiatives. Implications for practitioners are that clear responsibilities for the usability matters in development organizations have to be assigned. In addition, managers should be aware that people-oriented management is necessary to foster the awareness for usability in the organization. These insights complement earlier findings such as e.g. the need for a usability budget (Woywode et al. 2012).

Finally, some limitations of our research have to be mentioned. The explored data set was obtained in 2011. Hence, the knowledge of usability methods can be much more widespread today. Moreover, the responses to the questionnaire are dominated by managing directors of the software firms with a size of 6-10 employees. Hence, we have to acknowledge that the sample has an impact on the responses to the questions related to management support. Thus, related questions can only be interpreted as a self-reported measure of the status quo.

Our future research focuses on the diagnosing the status quo of usability methods in SD organizations along principles for user-centered and agile SD. Such a diagnosis is required in order to develop actionable recommendations for practitioners based on the already determined principles. For instance, we want to devise management instructions for implementing usability methods in SD teams. The perspective of the user organization on factors influencing the usability of enterprise systems should also be addressed.

\section{References}

Brault, C., Lykkegaard, B., \& Roques, B.-L. (2013). Ranking of the Top 100 European Software Vendor (pp. 1-7). Paris, FR.

Degarmo, T., Garland, P., \& Stuckey, D. A. (2011). The consumerization of IT: The next-generation CIO (pp. 1-26). Delaware, USA.

Doll, W. J. (1985). Avenues for Top Management Involvement in Successful MIS Development. MIS Quarterly, 9(1), 17-35.

European Union Commission. (2003). COMMISSION RECOMMENDATION - of 6 May 2003 concerning the definition of micro, small and medium-sized enterprises. Official Journal of the European Union, L 124, 36-41. 
Friedewald, M., Rombach, H. D., Stahl, P., Broy, M., Hartkopf, S., Kimpeler, S., ... Zoche, P. (2001). Softwareentwicklung in Deutschland - Eine Bestandsaufnahme. Informatik-Spektrum, 24(2), 8190.

Gemser, G., Jacobs, D., \& Ten Cate, R. (2006). Design and Competitive Advantage in TechnologyDriven Sectors: The Role of Usability and Aesthetics in Dutch IT Companies. Technology Analysis \& Strategic Management, 18(5), 561-580.

Harter, D. E., Krishnan, M. S., \& Slaughter, S. A. (2000). Effects of process maturity on quality, cycle time, and effort in software product development. Management Science, 46(4), 451-466.

International Organization for Standardization. (1998). ISO 9241-11: Ergonomic Requirements for Office Work with Visual Display Terminals (VDTs): Part 11: Guidance on Usability. Geneva, CH: International Organization for Standardization.

Kachigan, S. K. (1986). Statistical Analysis: An Interdisciplinary Introduction to Univariate and Multivariate Methods (p. 589). New York, NY, USA: Radius Press.

Mackay, D. J. C. (2003). Information Theory, Inference, and Learning Algorithms (p. 640). Cambridge, UK: Cambridge University Press.

Madan, A., \& Dubey, S. K. (2012). Usability Evalution Methods: A Literature Review. International Journal of Engineering Science \& ..., 4(2), 590-599.

Riemer, K., \& Vehring, N. (2010). IT'S NOT A PROPERTY! EXPLORING THE SOCIOMATERIALITY OF SOFTWARE USABILITY. In International Conference on Information Systems (p. Paper 215). St. Louis, MI, USA: AIS.

Scheiber, F., Wruk, D., Oberg, A., Britsch, J., Woywode, M., Maedche, A., ... Plach, M. (2012). Software Usability in Small and Medium Sized Enterprises in Germany: An Empirical Study. In A. Maedche, A. Botzenhardt, \& L. Neer (Eds.), Software for People (pp. 39-52). Berlin, DE: Springer.

Seffah, B. A., \& Metzker, E. (2004). The Obstacles and Myths of Usability and Software Engineering Avoiding the usability pitfalls involved in managing the software development life cycle. Communications of the ACM, 47(12), 71-76.

Simon, H. (2009). Hidden Champions of the Twenty-First Century: The Success Strategies of Unknown World Market Leaders (p. 402). Dordrecht, NL: Springer.

Woywode, M., Mädche, A., Wallach, D., \& Plach, M. (2012). Gebrauchstauglichkeit von Anwendungssoftware als Wettbewerbsfaktor für kleine und mittlere Unternehmen (KMU) : Abschlussbericht (p. 357). Mannheim, DE. 